\title{
Sieyès et le jury constitutionnaire : perspectives historico-juridiques
}

Sieyès and the constitutional jury: historical I judicial perspectives

\section{Marco Fioravanti}

\section{(2) OpenEdition \\ 1 Journals}

Édition électronique

URL : https://journals.openedition.org/ahrf/11225

DOI : 10.4000/ahrf.11225

ISSN : 1952-403X

\section{Éditeur :}

Armand Colin, Société des études robespierristes

\section{Édition imprimée}

Date de publication : 1 septembre 2007

Pagination : 87-103

ISSN : 0003-4436

\section{Référence électronique}

Marco Fioravanti, «Sieyès et le jury constitutionnaire : perspectives historico-juridiques », Annales historiques de la Révolution française [En ligne], 349 | juillet-septembre 2007, mis en ligne le 01 septembre 2010, consulté le 01 juillet 2021. URL : http://journals.openedition.org/ahrf/11225 ; DOI : https://doi.org/10.4000/ahrf.11225 


\title{
SIEYÈS ET LE JURY CONSTITUTIONNAIRE : PERSPECTIVES HISTORICO-JURIDIQUES*
}

\author{
Marco FIORAVANTI
}

\begin{abstract}
Cet article étudie l'origine du contrôle de constitutionnalité des lois en France, en particulier le projet de Sieyès présenté à la Convention nationale le 2 et le 18 thermidor an III - 20 juillet et 5 août 1795 - où aboutit l'idée du pouvoir constituant et de la séparation des pouvoirs. On cherche à démontrer que, dans une tradition française caractérisée par le refus de se doter des instruments pour garantir la constitution et limiter les pouvoirs du législateur, le projet de Sieyès est une tentative de créer un pouvoir neutre, par le jury constitutionnaire, organe politico-juridictionnel chargé soit de contrôler la constitutionnalité des lois, soit de proposer la révision de la constitution, soit d'exercer une fonction d'équité naturelle.
\end{abstract}

Mots-clés : Convention nationale, an III, Constitution de I'an III, pouvoir constituant, contrôle de constitutionnalité.

Tout le droit public français, à partir de la Révolution, est caractérisé par le refus de se doter d'institutions et de procédures de contrôle de la constitutionnalité de la législation. La loi, expression de la volonté générale, produit d'une assemblée législative souveraine, était soustraite à toute obligation de respecter des normes supérieures'. Le projet d'Emmanuel-

"Qu'il me soit permis d'exprimer mes remerciements à Monsieur Jean-Luc Bergounhou pour m'avoir aidé dans la traduction de l'article et aux rapporteurs des $A H R F$ pour les suggestions données.

(1) Ȧ propos du contrôle juridictionnel de la constitutionnalité des lois en France voir, en particulier, Ho Hю Ky, Le contróle de la constitutionnalité des lois en France, Paris, Pedone, 1926 ; Mario Battaglint, Contrihuti alla storia del controllo di costituzionalità delle leggi, Milan, Giuffrè, 1957 ; Mauro CAPPELLETT, Il controllo giudiziario di costituzionalità delle leggi nel diritto comparato, Milan, Giuffrè, 1968 ; Philippe SuEur. Histoire du droit public français $X V^{\top}-X V I I T$ siècle. La genèse de l'État contemporain. 2. Affirmation et crise de l'État sous l'Ancien Régime, Paris, PUF, 1989 ; Jcan-Louis MESTRE, «Juridictions judiciaires et contrôle de constitutionnalité en France de 1715 à 1814 ", dans Toward Comparative Law in the 2l" Century, Tokyo, Chuo University Press, 1998, p. 499-520 ; Alain LADUiEze, « Le contrôle de constitutionnalité de la loi vu par les penseurs libéraux français du $X X^{e}$ siècle ", dans Dominique CHacinolı.aud, (éd.), Aux origines du controle de constitutionnalité, $X V I I^{\prime}-X X^{\prime}$ siècle, Paris, LGDJ, 2003, p. 85-102 ; Didicr Maus, " La naissance du contrôle de constitutionnalité des lois en France ", dans L'esprit des institutions, l'équilibre des pouvoirs. Mélanges en l'honneur de Pierre Pactet, Paris, Dalloz, 2(003, p. 713 et s. ; Francesco Di Donato, "Il giudizio di costituzionalità nella Francia d'Ancien Régime : una storia politico-istituzionale. Parte prima. Fondamenti teorico-pratici. Parte seconda. Le dinamiche della prassi », Giomale di Storia costituzionale, IV (2004), n. 1, p. 147-165 et V (2005), n. 1, p. 65-86. 
Joseph Sieyès de l'an III fut une tentative d'introduire un contrôle de constitutionnalité par un organe institué à dessein et d'innover par rapport à la conception de la constitution et de la loi $^{2}$.

Un tournant radical sur le thème du contrôle de constitutionnalité des lois arriva en France seulement avec l'introduction du Conseil constitutionnel dans la constitution de $1958^{3}$.

Bien qu'une partie de la doctrine juridique ait soutenu que pendant l'Ancien Régime existait une sorte de contrôle de légalité des actes souverains aux lois fondamentales du royaume ${ }^{4}$, par les cours souveraines, le contrôle de constitutionnalité n'existait pas avant la Révolution. Par la suite, pendant la période révolutionnaire, la constitution n'était pas considérée comme une norme qui s'imposait sur les autres pouvoirs publics, mais était

(2) Sur la théoric constitutionnelle de Sicyès voir Yoeh KoUnc;, Théonie constitutionnelle de Sieyès, Préface de Joseph Barthelemy, Paris, Imprimeur Jouve, 1934 ; Paul Bastid, Les discours de Sieyès dans les débats constitutionnels de l'an III (2 et 18 thermidor). Paris, Hachette, 1939 ; Id., Sieyès et sa pensée, Paris, Hachette, 1939, éd. 1970 ; Colette ClavREuL, L'influence de la théorie d'Emmanuel Sicyès sur les origines de la représentation en droit public, Thèse, Paris I, 1982 ; Id., "Sieyès et la genèse de la représentation moderne ", Droits, III (1987), n. 6, p. 45 et s. ; Jean-Denis Bredin, Sieyès. La clé de la Révolution française, Paris, Éditions de Fallois, 1988 ; Stéphane Rıals, « Sieyès ou la délibération sans la prudence. Éléments pour une interprétation de la philosophie de la Révolution et de l'esprit du légicentrisme », Droits, VII (1991), n. 13, p. 123-138 ; Pasquale Pasouino, Sieyes et l'invention de la constitution en France, Paris, Odile Jacob, 1998 ; Andreï Tyrsenko, "Lordre politique chez Sieyès en l'an Ill ", $A H R F$, LXXVII (2000), n. 1, p. 27-45 ; Michel Troplr, "Sieyès et le jury constitutionnaire ", dans Michel Amzller, Philippe Ardant, JeanClaude Becane, et al. (éd.), La République. Mélanges en I'honneur de Pierre Avril, Paris, Montchrestien, 2001, p. 265-282; Lucien JAUME, "Sicyès et le sens du jury constitutionnaire : une reintérpretation », Droits, XVIII (2002), n. 36, p. 115-134. Michel TROPER, "La suprématie de la constitution et le jury constitutionnaire », dans Id., Terminer la Révolution. La Constitution de 1795, Paris, Fayard, 2006, p. 199-221.

(3) Sur le Conseil constitutionnel la bibliographie est très vaste, on se limite à renvoyer à une ouvrage de référence Pierre AVRIL, Le Conseil constitulionnel, Paris. Montchrestien, 2005 ; sur le légicentrisme de la culture juridique française et sur le sentiment de méfiance envers le juge, depuis le juge ordinaire jusqu'au juge constitutionnel, voir Fabrice HoURouEBIE, Sur l'emergence du contre-pouvoir juridictionnel sous la $V^{*}$ République, Bruxelles, Bruylant, 2004.

(4) Sur les lois fondamentales du royaume de France voir André LemaiRE, Les lois fondamentules de la monarchie française d'après les Théonciens de l'Ancien Régime, Paris, Fontemoing, 1907 ; Élie CARCassonne, Montesquieu et le problème de la Constitution française au XVIIr siècte, Paris, PUF, 1927 ; Bernard BASSE, La constitution de l'Ancienne France : principes et lois fondamentales de la royauté française, Liancourt, Presses Saint-Louis, 1973 ; Claire SAGUEZ-LovisI, Les lois fondumentales au XVIII siècle. Recherches sur la loi de dévolution de la couronne, Paris, PUF, 1984 ; Jean BARbEY. Frédéric Bl.UCHE, Stéphane Rials, Lois Fondamentales et succession de France, Paris, Duc, 1984 ; Jean BARBEY, « Genèsc et conservation des Lois fondamentales ", Droils, III (1986), p. 75-86; Paolo COMANDUCC1, “ Ordre ou norme? Quelques idées de constitution au XVIII'siècle ", dans Lucien JAUME, Michel Troper (éd.), 1789 et l'invention de la Constitution, Paris, LGDJ, 1994, p. 23-42 ; Mario CARAvale, Alle origini del dinito europeo. Ius comune, Droit commun, Common law nella dottrina giuridica della prima età moderna, Bologne, Monduzzi, 2005 , p. $156 \mathrm{et} \mathrm{s}$. Arnaud Vercine, La notion de constitution d'après les cours et assemblées à la fin de l'Ancien Régime (1750-1789), Paris, de Boccard, 2006. 
plutôt perçue comme un moyen de garantir l'organisation des pouvoirs et des fonctions.

Sieyès", après avoir donné, au début de la Révolution française, une contribution fondamentale à la théorie constitutionnelle comme théoricien du pouvoir constituant de la Nation française - c'est-à-dire du Tiers état et après avoir contribué à la rédaction de la Constitution du 3 septembre 1791, atténua son rôle politique et juridique durant la période suivante, jusqu'à disparaître pendant la Terreur.

C'est seulement après le 9 thermidor an II - 27 juillet 1794 - que Sieyès rentre sur la scène politique, en particulier avec deux discours importants à la Convention nationale, durant le nouveau débat constituant de l'an III, pendant lequel il proposa l'institution d'un organe chargé, entre autres choses, de contrôler la constitutionnalité des lois. Sieyès, en l'an III, avait comme priorité de trouver un moyen juridique pour terminer la Révolution, et le jury constitutionnaire en représenta un des instruments? La proposition du jury - son testament politique et juridique - apparaîtra comme l'aboutissement de sa théoric sur le caractère hiérarchiquement supérieur de la constitution.

Le projet que Sieyès présenta à la Convention en thermidor an III était le plus élaboré et le plus complet par rapport aux nombreux projets qui - durant la période précédente - furent présentés dans les assemblées révolutionnaires. Tout d'abord à l'Assemblée nationale constituante et après à la Convention nationale furent présentés un certain nombre de projets qui avaient prévu le contrôle de constitutionnalité des lois, de

(5) « La Révolution refusera toujours de confier à une instance extéricure au Corps législatif le soin de vérifier et de garantir le processus démocratique de la législation et la conformité des lois avec les principes posés dans la déclaration des droits de l'homme et du citoyen et dans lá constitution. Selon le légicentrisme dominant à l'époque, les principes se réalisent pleinement dans la loi voulue par le législateur ", Michel Perfú,, "La notion de constitution à la fin du $18^{\circ}$ sieccle ", dans Jacques Guithaumou, Raymonde MONNIER (éd.). Des notions-concepts en révolution. Autour de la libené politique à la fin du 18 siècle, Paris, Socićté des Études Robespierristes, 2003, p. 46; selon Didier Maus le contrôle de constitutionnalité des lois n’a jamais fait partie des valcurs fondatrices de l'histoire constitutionnelle française : «au nom de la souveraineté parlementaire, de son corollaire à propos du domaine illimité de la loi ct de l'ancestrale crainte révolutionnaire d'un contróle des tribunaux sur l'activité politique, tant la Cour de cassation que le Conscil d'État, ont toujours refusé d'entrer dans une quelconque logique d'un contrôle de la légalité constitutionnelle ", Didier MAUS, "Nouveaux regards sur le contróle de constitutionnalité par voie d'exception ", dans L'architecture du droit. Mélanges en lhonneur de Michel Troper, Paris, Economica, 2006, p. 665.

(6) À propos de l'orthographe du nom Sieyès voir Alhert MAтs!z: "Lorthographe du nom de Sieys ", $A H R F$, II (1925), p. 487; Albert Troux, "Lorthographe du nom de Sieyès ", AHRF, IX (1932), p. 66-67 ; Henri Calvet, "Sieys ou Sicyes", $A H R F, \mathrm{X}$ (1933), p. 538. On utilisera, comme a fait Paul Bastid, l'orthographe avec la deuxième $e$ accentuéc.

(7) "En l'an III, Sieyès était obsédé, encore plus peut-être que les autres thermidoriens par une seule idée : comment terminer définitivement la Révolution ? Conformément à son tour d'esprit, it la traduisait en termes constitutionnels : comment ćlaborer une constitution qui pourrait, en appliquant rigoureusement et habilement le système représentatif, verrouiller le système politique d'unc telle manière que la Révolution n’arrive plus à prendre un nouveau départ ? ", Bronislaw BAC 7KO, "Le contrat social des Français : Sieyès et Rousseau ", dans Id., Job, mon ami, Paris, Gallimard, 1997, p. 332-333 ; voir aussi $/ d$., Comment sortir de la Terreur? Thermidor et la Révolution, Paris, Gallimard, 1989. 
manière approximative et confuse. Par exemple, l'ouvrage de Brun de la Combe de $1789^{\circ}$ - Doutes sur les principes de M. l'Abbé Sieyès concernant la Constitution nationale - refusait la force illimitée du pouvoir constituant de Sieyès et proposa un pouvoir modérateur qui prévoyait les abus de l'autorité par rapport à la constitution. Le projet d'Isaac René Guy Le Chapelier, du 29 août $1791^{\prime \prime}$, représentait un exemple de la manière d'affronter le problème de la révision de la constitution. Le même jour, le député Pétion de Villeneuve présenta un projet" selon lequel la tâche de la révision de la constitution devait être confiée à des conventions nationales qui se réuniraient tout les vingt ans. Antoine Barnave, par contre, dans sa tentative de terminer la Révolution", dans un discours à la Constituante, le 31 août 1791 - Discours sur le pouvoir constituant, les Conventions nationales et le pouvoir de révision ${ }^{\prime 2}$ - refusa le système des conventions nationales et opta pour attribuer le pouvoir de révision au corps législatif qui réitérait son vote de révision par " la volonté répétée de quatre assemblées différentes $[\ldots]$ et ce vou aura été provoqué quatre fois par l'opinion publique, au moyen de l'élection des représentants $»^{13}$.

En outre, après la proposition de Barère qui - le 19 octobre 1792 invita tous les citoyens à présenter un projet de constitution pour participer, avec les conventionnels, au choix de la meilleure constitution pour la France, environ 300 projets parvinrent à la Convention ${ }^{14}$. Dans plusieurs projets on retrouva - sous des formes différentes et souvent désordonnées des organes chargés du contrôle de la constitutionnalité des lois.

La question des limites des pouvoirs du législateur était une question déjà connue des conventionnels. Le girondin Armand-Guy Kersaint avait proposé un tribunal de censeurs chargé de contrôler la conformité à

(8) Joseph-André BRUN dE LA COMBE, Réponse laconique aux "Observations sommaires " de $M$. l'abbé Syeiès [sic], sur les biens ecclésiastiques, \& doutes sur ses principes concernant la Constitution, Paris 1789.

(9) Pour le texte du projet voir Moniteur, Séance du 29 août 1791, p. 1005-1006.

(10) Pour le texte du projet voir Moniteur, Séance du 29 août 1791, p. 1008.

(11) Voir Patrice Gueniffey, «Terminer la Révolution : Barnave et la révision de la Constitution, auût 1791 ", dans François Furet, Mona Ozouf (éd.), Terminer la Révolution. Mounier et Barnave dans la Révolution française, Grenoble, PUG, 1988 ; Paolo VIOLA, Il trono vuoto. La transizione della sovranitá nella rivoluzione francese, Turin, Einaudi, 1989, en particulier la première partie Teminare la rivoluzione, p. 3-88 ; Timothy TACXETT, When the King Took Flight, Cambridge, Harvard University Press, 2003.

(12) Moniteur, Séance du 31 août 1791, p. 1020-1021.

(13) lbid., p. 1020

(14) Sur les projets présentés à la Convention en automne 1793 voir François GaLly, La notion de constitution dans les projets de constitution de 1793, Thèse, Paris, 1932 ; Michel PERTUE, " Les projets constitutionnels de 1793 ", dans Michel Vovel.t.F (éd.), Révolution et République. L'exception française, Paris, Kimé, 1994, p. 174-199 ; Nathalie OlLtvier, République et constitution en lan I, d'après les projets de 1793, Thèsc, Paris, 2002 ; Giuseppe G. FLoRIDIA, « Il governo repubblicano nei progetti dei Convenzionali : tableaux di ingegneria costituzionale ", dans Giomale di storia costituzionale, V (2005), n. 2, p. 107-130. 
la constitution de la conduite de la chambre et de l'exécutif ${ }^{15}$. Condorcet avait théorisé pour le contrôle des actes législatifs, un mécanisme complexe de démocratie directe ; il n'était pas complètement satisfait par le modèle simplement représentatif, pourtant il chercha des instruments pour protéger les citoyens du risque que le corps législatif viole la constitution. Un moyen était le veto populaire sur les lois ${ }^{16}$. La censure du peuple sur les actes législatifs dans le projet girondin était fondée sur un mécanisme qui prévoyait la dissolution de l'Assemblée si les assemblées primaires se prononçaient contre le décret ${ }^{17}$.

Le débat pour l'approbation de l'Acte constitutionnel montagnard du 24 juin 1793, fut également caractérisé par des propositions d'organes chargés de contrôler la constitutionnalité des actes du corps législatif. Le chapitre XV du projet de constitution jacobine contenait des dispositions qui instituaient un jury, $D u$ grand jury national ${ }^{1 \$}$. Maximilien Robespierre semblait favorable à un gardien des lois pour mettre un frein aux pouvoirs du législatif ${ }^{\prime 4}$. Marie-Jean Hérault de Séchelles, le plus important juriste jacobin, principal rédacteur de la constitution montagnarde, le 24 juin 1793, soutenait également que, malgré le fait qu'un jury national ait été rejeté, " on est resté d'accord qu'il fallait trouver un moyen de garantir le peuple de l'oppression du corps législatif $"^{2 n}$.

(15) Archives Parlementaires, I s., LXII, p. 420-429 ; voir aussi le projet de Charles Lambert, Archives Parlementaires, I s., LXII, p. 431 ; Rouzet, Archives Parlementaires, I s., LXII, p. 503 ss. ; Pressavin, Archives Parlementaires, I s., LXVII, p. 382-389 ; Baraillon, Archives Parlementaires, I s., LXVII, p. 187-220; A.-H. Wandelancourt, Archives Parlementaires, I s., LXVII, p. 417 ; P.-C.-F. Dupont, Archives Parlementaires, I s., LXVII, p. 314 ; Raffront du Trouillet, Archives Parlementaires, I s., LXVII, p. 139 et p. 390-391 ; David Williams, Archives Parlementaires, I s., LXIII, p. 583-591 ; Lagrange et Dupin, Archives Parlementaires, I s., LXIV, p. 248 ; Anonyme, Archives Parlementaires, I s., LXIII, p. 296-302; voir le projet de la section parisienne de l'Unité, 25 mai 1793, in Albert Soboul, Les sans-culontes parisiens et l'an II. Mouvement populaire et gouvernement révolutionnaire $(2$ juin $1793-9$ thermidor an 1I), Paris, Clavreuil, 1958, p. 116 et s.

(16) Titse VIII - De la censure du peuple sur les actes de la représentation nationale, et du droit de petition - du projet de constitution du 13 février 1793 ; voir J.-A.-N. Caritat de Condorcet, Au nom du comité de Constitution, donne lecture de son rapport contenant l'exposition des principes et des motifs du plan de ce comité sur le nouveau pacte social, dans Archives Parlementaires, I s., I.VIII, p. 588 et s.

(17) Selon Mathiez " pour masquer sa politique de classe d'un vernis de libéralisme démocratique en même temps que pour donner à ses partisans le moyen de paralyser une Assemblée legislative où domineraient ses adversaires, la Gironde inséra dans son projet de Constitution tout un système de référendum trẻs minutieusement réglé sous le titre de Censure du peuple sur les actes de la représentation nutionale ", Albert MATInEZ, "La Constitution de 1793 ", AHRF, V (1928), p. 497-521, publić dans Id., Girondins et Montagnards, Paris, Firmin-Didot, 1930, p. 95.

(18) "Art. I. L.c grand jury est institué pour garantir les citoyens de l'oppression du corps législatif et du conseil. Tout citoyen opprimé par un autre particulier a droit d'y rccourir. Il. La liste de jurés est composée d'un citoyen élu dans chaque département par les assemblées primaires. Le grand jury est renouvelé tous les ans, avec le corps législatif. III. Il n’applique point les peines, il renvoie devant les tribunaux. IV. Les noms de jurés sont déposés dans une urne au sein du corps législatif ", Moniteur, Séance du 16 juin 1793, p. 732.

(19) Ibidem.

(20) Moniteur, Séance du 24 juin 1793, p. 768. 
Le Balancier politique ${ }^{21}$, projet anonyme paru en l'an III, avait pour but d'intégrer à la constitution jacobine une institution qui garantissait le contrôle de constitutionnalité des actes du pouvoir législatif. Linstrument que l'auteur proposa pour limiter les pouvoirs du législateur était un Conseil de Sûreté publique " chargé spécialement de maintenir la séparation des pouvoirs $"{ }^{22}$. Selon le projet constitutionnel - Articles proposés pour la réforme de la Constitution de 1793 - le Conseil de Sûreté publique avait pour fonction de contrôler la conformité des décrets du corps législatif avec la constitution et la Déclaration des droits. Les décrets déclarés inconstitutionnels par le Conseil étaient validés par une sorte de référendum, procédure à caractère plus politique que juridique. Cette sanction indiquait la volonté de limiter le corps législatif en posant sur le même plan la constitution et la Déclaration des droits.

Les institutions de contrôle de constitutionnalité - prévues par les projets présentés à la Convention - étaient fondées sur une conception rousseauiste de la souveraineté et inspirées par la crainte de donner un trop grand pouvoir à un organe sans légitimité populaire ${ }^{23}$.

Le premier germinal an III - 22 mars 1795 - la Convention nomma la Commission des Sept, chargée d'élaborer les lois organiques pour l'application de la constitution de l'an I, commission composée par Sieyès, Merlin de Douai, Cambacérès, Mathieu, Thibaudeau, Lesage d'Eure-etLoir, et Creuzé-Latouche. Sieyès, dans le débat constitutionnel thermidorien, n'avait pas l'intention, comme la plupart des conventionnels, d'appliquer la constitution jacobine du 24 juin 1793. Avec l'insurrection des sans-culottes parisiens du $1^{\text {er }}$ prairial an III (20 mai 1795) - qui envahirent la salle de la Convention au cri « du pain et la constitution de 1793 »- la possibilité de promulguer des lois organiques pour l'application de la constitution montagnarde fut abandonnée $e^{24}$.

Le 29 germinal an III - 19 avril 1795 - la Convention nomma la Commission des Onze ${ }^{25}$, qui était convaincue de la nécessité d'écrire une constitution sans prendre en considération la possibilité de rédiger des lois organiques pour l'application de celle du 24 juin $1793^{26}$. Les rédacteurs de

(21) Le Balancier politique, Ou projet d'additions et de corrections à faire à la Constitution de 1793, Paris, chez Rondonneau, an III de la République.

(22) Ibid., p. 13.

(23) Voir Mario Battaglini, Contributi alla storia, op. cit., p. 59.

(24) Voir Paul Bastid, Sieyès et sa pensée, op. cit., p. 155.

(25) Antoine-Claire Thibaudeau, Louis-Marie La Révellière-Lépeaux, Denis-Toussaint Lesage d'Eure-et-Loir, François-Antoine Boissy d'Anglas, Jacques-Antoine Creuzé-Latouche, Jean-Baptiste Louvet, Théophile Berlier, Pierre-Claude-François Daunou, Jean-Denis Lanjuinais, Pierre-Toussaint Durand de Maillane. Pierre-Charles-Louis Baudin (des Ardennes).

(26) Sur la Commission des Onze voir Sergio Luzzatro, "Termidoro e costituzione : la Commissione degli undici ", Storia. Amministrazione. Costituzione. Annale Isap, II (1994), p. 55-76; Paolo Cоцомво, "La question du pouvoir exécutif dans l'évolution institutionnelle et le débat politique révolutionnaire ", AHRF, LXXVII (2000), n. 1, p. 1-26. 
la constitution de $1795^{27}$ considéraient la république comme étant menacée par deux dangers. Contre la menace de gauche et « la crainte qu'inspirait le faubourg Saint-Antoine ${ }^{28}$ fut prévu un système électoral censitaire et également la division du corps législatif en deux conseils. Contre la menace des royalistes fut établi un pouvoir exécutif directorial composé de cinq membres, c'est-à-dire un exécutif collégial comme l'était déjà le Comité de Salut public.

Sieyès, qui ne faisait pas partie de la Commission des Onze, se prononça contre le projet présenté à la Convention ${ }^{29}$ et proposa, quand les travaux de la commission furent quasiment terminés, un projet constitutionnel. Les organes prévus par Sieyès, dans son modèle de constitution, étaient au nombre de quatre : le Tribunat, chargé de proposer les lois, le Gouvernement chargé de leur exécution, la Législature qui devait décider de la promulgation de la loi proposée par le Tribunat et le Jury constitutionnaire, sa fonction principale étant de juger la constitutionnalité des lois. Mais la synthèse doctrinale qui précédait le projet de Sieyès était ambiguë30 et trop abstraite. « Les textes eux-mêmes, sibyllins à force d'être laconiques, demeuraient peu intelligibles pour les auditeurs. La déception de l'Assemblée fut réelle et rien n'était plus facile aux Onze que de l'exploiter ${ }^{31}$.

Les discours du 2 et du 18 thermidor an III - 20 juillet et 5 août 1795 représentaient une tentative de concilier le pouvoir constituant - établi par Sieyès en 1789 comme la force révolutionnaire même soustraite à toute loi

(27) Sur la constitution de l'an III, voir, parmi les autres, Albert MAthtez, Le Directoire, Paris, Colin, 1934 ; Georges LfFebvre, Le Directoire, Paris, CDU, 1943 ; Françoise Brunel, "Aux origines d'un parti de l'ordre : les projets de constitution de l'an III ", dans J. Nicol.AS (éd.), Mouvements populaires et conscience sociale, $X V V^{\prime}-X I X^{r}$ siècle, Paris, Presses du Palais royal, 1985, p. 687-696 ; Michel Perruf́, "Constitution de l'an III ", dans Albert Soboul, Dictionnaire historique de la Révolution française, Paris, PUF, 1989, p. 284-286; Michel Troper, "La Constitution de l'an III ou la continuité : la souveraineté populaire sous la Convention », dans Roger Dupux, Marcel Morabito (éd.), 1795. Pour une République sans Révolution, Rennes, PUR, 1996 ; J. BARI, J.-J. Clère, C. Courvoisiter, M. VerpauX (éd.), La constitution de l'an IIl ou l'ordre républicain, Dijon, EUD, 1998 ; G. ConAC, J.-P. MACHELON (éd.), La Constitution de l'an III. Boissy d'Anglas et la naissance du libéralisme constitutionnel, Paris, PUF, 1999 ; Sergio LUZzAITO, L'automne de la Révolution. Luttes et cultures politiques dans la France thermidorienne, Paris, Champion, $2(x) 1$; Miche! Troper, Terminer la Révolution, op. cit.

(28) Paul Bastid, Sicyès ét sa pensée, op. cif., p. 170 ; voir Raymonde MonNik̂, Le Faubourg SaintAntoine (1789-1815), Paris, Société des Études Robespierristes, 1981.

(29) « Lorsqu'il faut donner une nouvelle constitution au pays, en l'an III, c'est ainsi un Sieyès inchangé qui prend la parole, le 2 thermidor an III, un maitre non pas dégrisé mais encore affermi dans ses certitudes par les malheureuses expériences antérieures (les expériences des autres, pense-t-il sans doute, non sans quelques motifs dont la portée, simplement, est douteuse), maniant avec hauteur le crayon rouge pour censurer le projet en discussion et - tout en prétendant ne présenter qu'une "opinion" - recourant sans nuances au vocabulaire de la vérité, de la nécessité et opposant la force de la " chose "à la " magie des mots" ", Stéphane RIALS, « Sieyès ou la délibération sans la prudence ", op. cit., p. 131.

(30) “Son propos est un monument d'ambiguïté - un concentré des ambiguïtés thermidoriennes, qu'il reproduit et redouble à un niveau supéricur en s'efforçant de les trancher », Marcel GaUCHET, $L a$ Révolution des pouvoirs. La souveraineté, le peuple et la représentation 1789-1799, Paris, Gallimard, 1995, p. 160.

(31) Paul BASTID, Sieyès et sa pensée, op. cil., p. 171. 
- avec le pouvoir de contrôle de la constitution, au moyen d'une autorité prévue par le texte constitutionnel, située à un niveau plus élevé par rapport aux autres. « Lappel au pouvoir constituant préfigure dès 1789 la création du jury constitutionnaire que Sieyès proposera en l'an III ${ }^{32}$. Lintuition $^{33}$ de Sieyès était de garantir la conformité des lois avec la constitution et de protéger les droits des citoyens face au législateur même. Le choix d'un jury constitutionnaire représentait l'issue du raisonnement commencé à partir de 1789 sur la division entre pouvoir constituant et pouvoirs constitués.

Le 2 thermidor an III, Sieyès présenta ses idées sur le gouvernement et sur la constitution politique :

" En fait de gouvernement, et plus généralement en fait de constitution politique, unité toute seule est despotisme, division toute seule est anarchie. Division avec unité donne la garantie sociale, sans laquelle toute liberté n'est que précaire. [...] Divisez, pour empêcher le despotisme ; centraliser, pour éviter l'anarchie. [...] Je ne connais que deux systèmes de division des pouvoirs : le système de l'équilibre et celui du concours, ou, en termes à-peuprès semblables, le système des contrepoids et celui de l'unité organisée " $^{34}$.

Dans cette intervention, Sieyès - qui préférait le système de l'unité organisée plutôt que les contre-pouvoirs anglais - reprit ses théories en faveur du système représentatif ${ }^{35}$, le meilleur pour gouverner un pays comme la France. Sieyès critiqua la position - d'inspiration démocratique et jacobine - de ceux qui, comme Hérault de Séchelles et Robespierre, voyaient le système représentatif comme alternatif à la démocratie. Il critiqua ce principe avec l'exemple célèbre, quoique pittoresque ${ }^{36}$, de la poste :

(32) Christine Fauré, Introduction à des manuscrits de Sieyès 1773-1799, Paris, Champion, 1999, p. 29.

(33) Durand de Maillane avait proposé. également, un jury constitutionnaire : "Je n'eus que l'idée d'un jury constitutionnel. Après avoir proposé cette institution, je n'y donnais pas de suite. Peut-être aurait-elle mieux réussi dans cette commission [commission des Onze] que dans la Convention où elle fut présentée et soutenue par M. Sieyes. Si on l'cût adoptée, le Directoire n'aurait pas foulé la république à ses pieds, ainsi que les législateurs ; il aurait eu un surveillant qui l'eút fait marcher dans la ligne de ses devoirs sans empiéter sur les droits du corps législatif, sans insulter et aux lois et à leurs auteurs. J'ai lieu de croire que le jury constitutionnel proposé par M. Sieyes n'était pas assez simple ; la complication d'un tribunat, d'un gouvernement, d'une législature, d'un jury constitutionnaire le fit rejeter, tandis que, réduit ou borné à un scul jury constitutionnel, on l'eut probablement approuvé. Il suffisait pour tenir toutes les premières autorités en respect et dans les bornes de leurs fonctions. Mon idée était aussi de le composer de vingt-quatre membres choisis parmi les hommes les plus instruits et en même temps les plus attachés à la chose publique et à la constitution : on eût placé un ou deux procureurs-généraux auprès de ce jury, afin de relever et de poursuivre tous les écarts contraires à l'ordre public et constitutionnel de l'État », Pierre-Toussaint Durand de Maillane, Histoire de la Convention nationale, Paris, Baudouin, 1825, p. 275-276.

(34) Moniteur, Séance du 2 thermidor an III, p. 1236.

(35) Voir Colette Clavreul, L'influence de la théorie d'Emmanuel Sieyès, op. cit., en particulier Contrôle de constitutionnalité, p. 155-165.

(36) Lexpression est de Georges LEsCUYER, Histoire des idées politiques, Paris, Dalloz, 2001, p. 355. 
«C'est comme si l'on voulait prouver aux citoyens qui ont besoin d'écrire, à Bordeaux, par exemple, qu'ils conserveront bien mieux toute leur liberté, s'ils veulent se réserver le droit de porter leurs lettres eux-mêmes, car ils le peuvent, au lieu d'en confier le soin à cette partie de l'établissement public qui en est chargée. Peut-on voir, dans un si mauvais calcul, les véritables principes ? $"{ }^{37}$. Après ces considérations sur le gouvernement et sur la constitution, il demanda l'institution du jury constitutionnaire, organe plus politique que juridictionnel : "C'est un véritable corps de représentants que je demande, avec mission spéciale de juger les réclamations contre toute atteinte qui serait portée à la constitution ${ }^{38}$.

Mais surtout dans le discours du 18 thermidor an III, Sieyès exposa les principes concernant un organe de contrôle de l'activité du législateur. Le 18 thermidor, le Moniteur reporte l'opinion de Sieyès sur les attributions et l'organisation du jury constitutionnaire, jury organisé selon 17 articles $^{39}$ et chargé de contrôler le respect de la constitution ${ }^{40}$.

"La nécessité d'un jury de constitution - soutenait Sieyès à la Convention nationale - forme une question en quelque sorte préliminaire ; elle n'a pas souffert de difficulté. Comment en effet la prévoyance du législateur s'accoutumerait-elle à l'idée d'une constitution abandonnée, pour ainsi dire, à elle-même au moment de sa naissance ? Une constitution est un corps de lois obligatoires, ou ce n'est rien ; si c'est un corps de lois, on se demande où sera le gardien, où sera la magistrature de ce code ${ }^{44}$.

Le jury constitutionnaire, « dépositaire conservateur de l'acte constitutionnel ", aurait été composé par 108 membres, et aurait été renouvelé par tiers chaque année, à la même époque que le corps législatif. Le premier jury aurait été constitué par la Convention au moyen d'un scrutin secret, de manière à ce qu'un tiers des membres fut choisi parmi ceux de la Constituante, un autre tiers parmi ceux de la Législative, et le dernier tiers parmi les membres de la Convention. Ensuite, l'élection d'un tiers ou des

(37) Moniteur, Sćance du 2 thermidor an III, p. 1236.

(38) lbid., p. 1237.

(39) « Je demande d'abord un jury de constitution, ou, pour franciser un peu plus le mot de jury, et le distinguer dans le son de celui de juré, une jury constitutionnaire ", Opinion de Sieyès, sur plusieurs articles des titres IV et $V$ du projet de Constitution, prononcée à la Convention le 2 thermidor de l'an troisieme de la République, Paris an III, p. 7. Sur la langue et sur l'invention d'un nouveau langage politique par Sicyc̀s voir Jacques Gulluaumov, Sieyès el l'ordre de la langue. L'invention de la politique moderne. Paris, Kimé, 2002 : "Précisant en l'an III sa conception de la Constitution et du gouvernement, Sicyès nous donne de nouveaux éléments pour mieux cerner cette relation éminemment normative du législateur à la " langue politique " ", p. 132.

(40) Opinion de Sicyès sur les attributions et l'organisation du jury constitutionnaire proposé le 2 thermidor, prononcée à la Convention nationale le 18 du même, lan III de la République, dans Moniteur, Séance du 18 thermidor an Ill, p. 1311.

(41) lbidem. 
36 entrants aurait été réalisée par le jury lui-même parmi les 250 membres qui auraient dû, à la même époque, sortir de l'un ou l'autre des deux conseils du corps législatif ${ }^{42}$. "Cette volonté d'assurer la pérennité politique d'une notabilité révolutionnaire se retrouvera chez l'"oracle de la science politique" bien au-delà de l'an III $~^{43}$. Le jury constitutionnaire était l'expression d'une vision particulière de la séparation des pouvoirs ${ }^{44}$.

Le jury aurait exercé trois fonctions. Garantir la sauvegarde de la constitution en fonctionnant comme un tribunal de cassation de l'ordre constitutionnel, un jury de cassation $»^{45}$. Perfectionner la constitution, en étant un lieu désigné pour présenter les projets de révision constitutionnelle, un « jury de proposition $»^{46}$. La troisième prérogative du jury est la plus originale : il aurait exercé un contrôle sur les jugements émis par la juridiction ordinaire fondé sur le droit naturel.

La première fonction de contrôle de constitutionnalitét ${ }^{47}$ est très complexe. Les actes sur lesquels se serait prononcé le jury étaient : les actes du Conseil des Cinq-Cents et du Conseil des Anciens, les actes inconstitutionnels en matière électorale, les actes inconstitutionnels des assemblées primaires et les actes du Tribunal de cassation. "Les actes déclarés inconstitutionnels par arrêt du jury constitutionnaire - article VIII du projet sont nuls et comme non avenus $"^{4 k}$.

En tout cas, le jury ne pouvait jamais déclarer des actes anticonstitutionnels de sa propre initiative, mais seulement en cas de dénonciation par le Conseil des Anciens, par le Conseil des Cinq-Cents ou par tout citoyen. Sieyès fut le premier en France qui conçut et proposa « un système achevé de contrôle de la Constitution et de maintien des principes fondamentaux de la société ${ }^{44}$. Le jury exerçait les fonctions de tribunal de cassation de l'ordre constitutionnel, en annulant les actes contraires à la loi fondamentale. Selon Paul Bastid, le projet de Sieyès, exposé dans l'Opinion du 18 thermidor, sur le contrôle de constitutionnalité des lois présentait « un incontestable désordre ${ }^{\text {sil }}$ et Michel Troper pense que ce désordre est

(42) Idid., p. 1315-1316. 2002, p. 117

(43) Marcel MoRaBrTo, Histoire constitutionnelle de la France (1789-1958), Paris, Montchrestien,

(44) V. Pasquale Pasouino, Sieyes et l'imvention, op. cit., p. 13.

(45) Moniteur, Séance du 18 thermidor an IIl, p. 1311.

(46) Ibidem.

(47) Pasquino considère le jury constitutionnaire de Sieyès comme le prédécesseur du contrôle de constitutionnalité des lois, par contre, selon Michel Troper - contre la thèse majoritaire en doctrine (Paul Bastid, Marcel Gauchet, Colette Clavreul, Jean-Denis Bredin) - le jury constitutionnairc, thćorisć par Sieyès en thermidor an III, n'était pas le prédécesseur du contrôle de constitutionnalité des lois : " cette institution est néanmoins fort différente des cours constitutionnelles que nous connaissons aujourd hui», Michel Troper. "Sieyés et le jury constitutionnaire ", op. cit., p. 272 ; du méme avis Maurizio Fioravant, Costituzione, Bologne, II Mulino, 1999, p. 117.

(48) Voir Mario Bajtaglini, Contributi alla storia, op. cit., p. 62.

(49) Jean-Denis BrLdin, Sieyes, op. cit., p. 367.

(50) Paul Bastid, Sieyès el sa pensée, op. cit., p. 436. 
démontré par le fait que les actes sur lesquels le jury se prononce ne sont pas des lois, mais des actes de l'un ou de l'autre des conseils, et non les actes législatifs qui devaient être nécessairement actes des deux chambres ou des deux sections du corps législatif. En fait, le premier alinéa de l'article VI du projet de Sieyès prévoyait que « le jury constitutionnaire [se prononcerait] sur les violations ou atteintes faites à la constitution, qui lui seraient dénoncées, contre les actes, soit du Conseil des Anciens, soit du Conseil des Cinq-Cents ». Dans ce cas-là, selon Troper, « les cas d'inconstitutionnalité seront rares et d'ailleurs difficiles à imaginer ${ }^{51}$. En outre, la Constitution de l'an III avait prévu des garanties internes pour se protéger : tout d'abord était prévue la séparation entre les conseils et le pouvoir exécutif et ensuite le Conseil des Anciens pouvait exercer un contrôle sur la constitutionnalité en refusant d'approuver des actes pris par le Conseil des Cinq-Cents sans respecter les formes et les procédures prévues par la constitution (article 88).

Dans le cadre de la deuxième fonction, à savoir le rôle de proposition de réformes constitutionnelles - fonction non prévue par les conseils constitutionnels d'aujourd'hui - le jury constitutionnaire n'était pas seulement le "gardien et le défenseur de la constitution $"^{52}$, mais avait également la tâche d'améliorer et de perfectionner, soit la constitution, soit la Déclaration des droits de l'homme, par rapport aux nouvelles exigences et aux changements de la sociétés ${ }^{53}$. C'était un moyen qu'avaient les révolutionnaires pour mettre un frein à la Révolution et pour contrôler un pouvoir sans contraintes, comme le pouvoir constituant. En 1789 le pouvoir constituant, représenté par la Nation française, était illimité :

« De quelque manière qu'une nation veuille, - écrivait Sieyès dans son Qu'est-ce que le Tiers-état? - il suffit qu'elle veuille; toutes les formes sont bonnes, et sa volonté est toujours la loi suprême. [...] Répétons-le : une nation cst indépendante de toute forme; et de quelque manière qu'elle veuille, il suffit que sa volonté paraisse, pour que tout droit positif cesse devant elle, comme

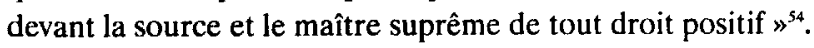

En 1795 , le pouvoir constituant était discipliné de manière restrictive dans les fonctions du jury : « Le pouvoir constituant, reconnu [par Sieyès] à la nation en 1789, alors inconditionné et illimité, devient - selon Michel Pertué - réglé et de plus en plus restreint dans les mains du jury constitu-

(51) Michel Troper, "Sieyès et le jury constitutionnaire ", op. cit., p. 273.

(52) Selon l'expression connue de Carl SchmiTt, Der Hüter der Verfassung, Tubingen, Mohr, 1931.

(53) Voir Vida AzımI, "Limpossible révision de la Constitution : l'exemple de l'an III ", dans Jean Bart, Jean-Jacques Clekre, Claude Courvoisier, Michel Verpaux (éd.), La constitution de l'an III, op. cit., p. 199-211.

(54) Joseph-Emmanuel SıEYĖs, Qu'est-ce que le Tiers-État ?, Paris, SHRF, 1888, p. 69-70. 
tionnaire de l'an III, puis du collège des conservateurs de l'an VIII ${ }^{55}$. Le pouvoir constituant agissait selon les normes du droit naturel, par contre le pouvoir constitué selon les normes du droit positif $f^{\text {st }}$. Le projet de Sieyès de l'an III fut également une tentative de résoudre l'antithèse entre démocratie directe et démocratie représentative, entre souveraineté du pouvoir constituant et souveraineté des pouvoirs constitués ${ }^{57}$. Comme la Révolution avait donné, selon Sieyès, ses résultats, il faudrait éviter à l'avenir de retourner à des assemblées constituantes ou à des conventions et il était nécessaire de prévoir une révision périodique de la constitution par la constitution même. Le pouvoir appartenait toujours au peuple, mais il s'agissait d'un pouvoir réglementé.

Ce à quoi avait déjà pensé Barnave et Condorcet, était proposé par Sieyès avec de nouveaux instruments. Le pouvoir constituant appartenait uniquement aux assemblées primaires, c'est-à-dire au peuple souverain. En revanche, le pouvoir de révision constitutionnelle appartenait, dans le projet de Sieyès, au jury constitutionnaire. Le 18 thermidor an III, Sieyès dans le sillage d'un débat sur les droits des générations futures qui avait caractérisé la fin du XVIII' siècle et impliqué plusieurs révolutionnaires, en particulier Condorcet ${ }^{58}$ - soutenait :

"Sans vouloir disputer aux générations futures le droit de faire à cet égard tout ce qui leur conviendra, il est permis, et c'est encore un devoir de remarquer que les véritables rapports d'une constitution politique sont avec la nation qui reste, plutôt qu'avec telle génération qui passe ; avec les besoins de la nature humaine, communs à tous, plutôt qu'avec des différences individuelles. Ces considérations nous font une loi de donner à notre acte constitutionnel, ainsi qu'on vient de le dire, un principe de perfectionnement

(55) Michel PERTUE, Aux origines du gouvemement révolutionnaire (février 1793-mars 1793). Démocratie et dictature en révolution, Thèse, Paris II, 1976, p. 417.

(56) Sur le pouvoir constituant voir Lucien JAUME, * II potere costituente in Francia dal 1789 a De Gaulle ", dans Paolo Pombeni (éd.), Potere costituente e riforme costituzionali, Bologne, Il Mulino, 1992, p. 33-51 ; Maurizio Fioravanti, “ Potere costituente e diritto pubblico ", dans Id., Stato e costituzione. Materiali per una storia delle dottrine costituzionali, Turin, Giappichelli, 1993, p. 220 et s. ; Claude KLEIN, Théorie et pratique du pouvoir constituant, Paris, PUF, 1996; Antonio NEGRI, Le pouvoir constiruant. Essai sur les alternatives de la modernité, Paris, PUF, 1997 ; Ernst-Wolfgang BÖCKFNFÖRDE, Le droit, l'État et la constitution démocratique, Paris, LGDJ, 2000 ; Michele SurDI, Faida, contratto, patto. Studi sulla capacità costituente, Turin, Giappichelli, 2001 : Michel TrOPER, « Le projet de Constitution curopéenne et la question du pouvoir constituant ", Cité, XIII (2003), p. 101-111 ; Arnaud LE PILLOULR, Les pouvoirs non-constituants des Assemblées constituantes. Essai sur le powvoir instituant, Préface de Michel Troper, Paris, Dalloz, 2005 ; Olivier CAYL.A, "L'obscure théorie du pouvoir constituant originairc ou l'illusion d'une identité souveraine inaltérable ", dans L'architecture du droit, op. cit., p. 249-265 ; Pietro Giuseppe Grasso, /l potere costituente, Turin, Giappichelli, 2006.

(57) Maurizio FioRavanti, Appunti di storia delle costituzioni moderne. Le libenta fondamentali, Turin, Giappichelli, 1995, p. 65.

(58) Voir Paola Persano, "L'Encyclopédie fra continuità e mutamento », Giomale di Storia costituzionale, Il (2002), n. 4, p. 69-80 ; Id., « Tempo, rivoluzione, costituzione: un bilancio storiografico , Storica, XI (2005), n. 31, p. 45-75. 
illimité, qui puisse le plier, l'accommoder aux nécessités de chaque époque, plutôt qu'une faculté de reproduction ou de destruction totale, abandonnée au hasard des événements ${ }^{54}$.

Mais le jury ne pouvait pas intervenir directement sur la réforme de la constitution ; il avait seulement le rôle de proposition. Pour éviter des émeutes et des soulèvements populaires dans une période de réforme de la constitution, le jury, chaque dix ans, devait présenter aux conseils législatifs et aux assemblées primaires un cahier ou projet d'amélioration de la constitution. Les assemblées primaires - réunies chaque année pour l'élection des représentants du peuple - se seraient prononcées en faveur ou contre la possibilité de déléguer un pouvoir constituant temporaire à la législature actuelle $^{(0)}$. Si la majorité était contraire, le processus de révision s'interrompait pour dix ans, après lesquels le jury aurait eu la possibilité de représenter un projet de révision. Mais, selon Paul Bastid, " il est difficile de concevoir un contrôle du Jury constitutionnaire, autorité constituée, s'exerçant sur le pouvoir constituant lui-même, considéré dans son originalité intrinsèque ${ }^{\prime \prime \prime}$.

La troisième fonction du jury était un contrôle d'équité - fondé sur le droit naturel - sur les jugements des juridictions ordinaires. Le projet prévoyait que : "art. XIV. Chaque année, le dixième au moins des membres du jury constitutionnaire, pris au sort, se [formerait] en jury d'équité naturelle. Cette section [serait], en sus des deux attributions précédentes, exclusivement chargée de prononcer sur les demandes officielles qui lui seraient portées par les divers tribunaux, à l'effet d'avoir un arrêt d'équité naturelle sur les cas qu'ils déclareraient n'avoir pu juger, faute de loi positive qui pût s'y appliquer, ou ne pouvoir juger que contre leur conscience, d'après le texte seul de la loi. XV. Les arrêts d'équité naturelle seront exécutés par le tribunal qui aura formé la demande officielle, ou par tout autre, au choix du jury constitutionnaire. XVI. Les arrêts d'équité naturelle seront officicllement communiqués dans le mois au Conseil des Cinq-Cents. Le jury constitutionnaire ne peut rendre aucun arrêt du propre mouvement ».

Le jury aurait exercé les fonctions de tribunal d'équité naturelle - un tribunal des droits de l'homme $\mathrm{e}^{\mathrm{k} 2}$ - au cas où les tribunaux ordinaires considéreraient que la loi positive avait des lacunes ou était injuste. Selon Michel Troper, « il ne s'agira, comme le prétend Sieyès, d'une simple application de principes d'équité naturelle qui seraient objectifs et connaissa-

(59) Moniteur, Séance du 18 thermidor an HI, p. 1311.

(60) "Dans ce système, à qui appartient vraiment le pouvoir de révision ? [...] Il ne s'agit là que d'un pouvoir constituant amoindri, conditionné à la fois dans son apparition, dans son objet ct dans sa durée ", Paul BastID, Sicyés et sa pensée, op. cit., p. 441-442.

(61) Ibid., p. 437.

(62) L'expression cst de Colette CLAVREUL, L'influence de la théonie d'Emmanuel Sieyès, op. cit., p. 160. 
bles, mais de l'exercice d'une volonté et plusieurs orateurs dénonceront le caractère vague de l'équité naturelle, c'est-à-dire le pouvoir excessif, voire arbitraire du jury $»^{n 3}$.

Au cas où la loi serait imparfaite, une intervention était nécessaire pour perfectionner le droit en vigueur. Sieyès, qui s'inspirait de la common law anglaise, avait pensé à ce dispositif pour aider les juges s'ils se trouvaient en face d'un texte législatif manquant de clarté ou « injuste». Dans ce cas-là, les tribunaux devaient recourir au jury comme tribunal supérieur d'équité. Mais cette fonction contredit le sens du contrôle de constitutionnalité et le sens de la constitution même. La présence d'une constitution écrite doit nécessairement prévoir que les lois lui soient conformes et pourtant est nécessaire un organe chargé de faire respecter ce principe. Mais cet organe doit se limiter à faire respecter la constitution positive et pas le droit naturel, qui est aussi sujet à plusicurs interprétations et conceptions. Selon Marcel Gauchet, l'équité naturelle apparaît « comme une pièce surajoutée, sans lien nécessaire avec les autres éléments ni surtout avec la finalité principale du dispositif. Elle heurte de surcroît la philosophie de l'égalité devant une loi rigoureusement générale, devenue comme une seconde nature pour le personnel révolutionnaire, et dont Sieyès, en son temps, avait été l'un des plus illustres chantres. Pis même, elle réveille le spectre des " jurisprudences arbitraires" d'Ancien Régime, et de leurs cohortes honnies " de commentateurs et d'interprétateurs " ${ }^{(44}$.

Si on prévoyait la possibilité d'un recours au droit naturel, la constitution cesserait d'être la loi suprême de l'État. "On voit par là que le jury constitutionnaire est pour lui une institution comparable à un législateur et non à un tribunal. Tandis que le tribunal ne fait qu'appliquer une règle préexistante sans pouvoir exprimer une volonté, sans être arbitraire $"$ ts.

Pour contrer le projet de Sieyès, intervinrent à la Convention Antoine-Claire Thibaudeau, Joseph Eschassériaux, Louis-Marie La Révellière-Lépeaux, Denis-Toussaint Lesage, Jean-Baptiste Louvet. Selon Louvet - le 30 thermidor an III - le contrôle de constitutionnalité était déjà présent dans la constitution même. Les mécanismes constitutionnels prévus par le projet de constitution, présenté par la Commission des Onze, prévoyaient un contrôle interne de la constitutionnalité des lois par l'obligation « imposée au pouvoir exécutif d'annuler les actes de ses sous-ordres qui y seraient contraires et à l'égard de cette obligation il est responsable, comme il l'est pour les infractions qu'il pourrait commettre lui même à l'acte constitutionnel " ${ }^{\text {th }}$. Selon une critique, déjà soulevée par Thibaudeau le 24 thermidor an III $^{67}$ - qui aurait contrôlé les gardiens de la constitution?

(63) Michel Troper, " Sieyès et le jury constitutionnaire ", op. cit., p. 281.

(64) Marcel Gauchet, La Révolution des pouvoirs, op. cit., p. 179.

(65) Michel Troper, « Sieyès et le jury constitutionnaire », op. cit., p. 276.

(66) Moniteur, Séance du 30 thermidor an III, p. 1328. 


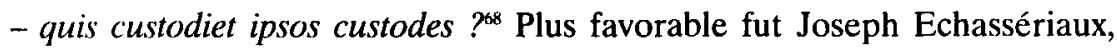
mais il refusait la fonction d'équité naturelle, « inutile dans notre système de législation civile, et dangereux en politique $\aleph^{\prime \prime \prime}$. Le jury provoqua les craintes des conventionnels thermidoriens, pétris de la leçon de Rousseau et donc rétifs à accepter un pouvoir supérieur au pouvoir de l'assemblée, la seule légitimée à représenter la volonté générale. Les conventionnels n'aimaient point à établir un pouvoir de contrôle supérieur à celui des assemblées législatives. « Plaidant en faveur de la prédominance de la loi, - écrit Marcel Morabito - l'exaltation révolutionnaire de la représentation demeurait incompatible avec toute idée de contrôle de constitutionnalité ${ }^{70}$.

La proposition d'instituer un jury constitutionnaire par Sieyès contribua - au-delà du contrôle de la constitutionnalité des lois - de manière originale à un passage de la conception de la constitution comme mécanisme, comme une simple distribution des pouvoirs, à la constitution comme norme obligatoire, supérieure à toutes les autres ${ }^{71}$. La conséquence de la «super légalité " $^{22}$ de la constitution est la possibilité d'annuler les actes contraires à elle ${ }^{73}$.

Linstitution d'un jury constitutionnaire - réduite à néant dans le débat constitutionnel de l'an III - trouva son application dans la constitution de l'an VIII sous la forme du Collège de conservateurs, ensuite Sénat, qui a priori était l'organe le plus haut dans la hiérarchie prévue par la constitution de $1799^{74}$.

(67) Moniteur, Sćance du 24 thermidor an III, p. 1239 ; Thibaudeau, à propos du jury constitutionnaire, soutenait : "Sicyes avait refusé de prendre part à nos travaux ; la Convention en avait déjà adopté une partie. Je parlai donc après lui, cependant je m'expliquai avec ménagement. J'essayai de prouver que son plan rentrait à beaucoup d'égards dans le nôtré, et j'en demandai le renvoi à la commission des onze, sans que la discussion fût pour cela interrompue. Ces propositions furent adoptées. Sieyes avait imaginé, sous le nom de Jurie constitutionnaire, un corps de censcurs qui, supćricur à tous les pouvoirs, devait preserver la constitution de toute atteinte et y proposer des réformes. Cette institution fut la scule partic de son projet prise en consideration. La commission voulut l'accommoder à son plan de constitution et la proposa à la Convention. La jurie y trouva des défenseurs et des adversaires. Je la combattis (le 24 thermidor), et elle fut rejetée à la presqu'unanimité. Elle me parut une superfétation inutile et dangereuse ", AntoineClaire Thibaudeau, Mémoires sur la Convention et le Directoire, t. 1" Convention, Paris, Baudoin, 1824, p. 186.

(68) Thibaudeau disait : " Ainsi chez un peuple des Indes, la croyance vulgairc est, dit-on, que le monde est porté par un éléphant, et cet ćléphant par une tortue ; mais quand on vient demander sur quoi repose la torture, adieu l'érudition ", dans Moniteu, Sćance du 30 thermidor an III, p. 1330.

(69) Moniteur, Séance du 30 thermidor an III, p. 1329.

(70) Marcel Mokabito, Histoire constitutionnelle, op. cit., p. 118.

(71) Le jury constitutionnaire exerçait une "garantie de la légalité du système de normes", Colette Clavreul, L influence de la théorie d'Emmanuel Sieyès, op. cit., p. 163.

(72) Pasquale Pasouino, Sieyès et l'invention, op. cit., p. 12.

(73) V. Apostolos Papatolias, Conception mécaniste et conception normative de la constitution, Paris, LGDJ, 2002.

(74) Sur la constitution de l'an VIIl voir Jean BOURDON, La constitution de l'an VIII, Rodez, Carrère, 1942 ; Félix PONTEIL, "Le régime autoritaire et les grandes lois organiques de l'an VIII ", Revue intemationale d'histoire politique et constitutionnelle, IV n.s. (1954), p. 226-243; Michel PERTUE, "La constitution de l'an VIII ", dans Albert Soboul, Dictionnaire historique, op. cit., p. 286-287 ; Pierre-Clément Timbal, André Castaldo, Histoire des institutions publiques et des faits sociaux, Paris, Dalloz, 2000, p. 521-525. 
Refusé en l'an III, le projet de Sieyès fut adopté en l'an VIII par deux commissions, mais considérablement modifié. Le Collège des conservateurs, devenu plus tard le Sénat conservateur, était composé des 80 membres ; pour être sénateur il fallait avoir quarante ans; le choix des membres était moins libre car le Sénat était obligé de choisir entre trois candidats présentés, le premier par le Corps législatif, le deuxième par le Tribunat et le troisième par le premier consul ; de plus, si le même candidat était présenté par les trois autorités, le Sénat était obligé d'accepter. La constitution de l'an VIII fut un texte qui remplaça la centralité du pouvoir législatif - caractéristique de la tradition révolutionnaire ${ }^{7 s}$ - par le primat de la fonction gouvernementale et, dans la critique de la centralité du législateur, apparait la question chère à Sieyès d'un contrôle des actes du législatif. Dans ce cas-là, le contrôle de constitutionnalité avait une valeur plus politique que juridictionnelle par rapport à l'an III. “ Considéré sous son aspect politique, le Jury ou le Collège représentait une sorte de grande Académie révolutionnaire, où les traditions de 1789 devaient se voir pieusement conservées et entretenues ${ }^{76}$. Le refus de Sieyès du mot « jury » pour utiliser «Collège » et puis «Sénat » indique une spoliation de la valeur juridictionnelle de cet organe en faveur d'un sens plus politique ${ }^{77}$.

La solution trouvée en France, quand on a cherché à introduire un contrôle de la conformité des lois ordinaires par rapport à la norme constitutionnelle, fut toujours de confier le contrôle à un organe de nature politique et cela pour deux raisons, l'une historique et l'autre idéologique. La première raison vient de l'hostilité que l'on avait en France vis-à-vis des interférences du pouvoir judiciaire avec les autres pouvoirs ; la seconde raison est liée à l'influence des doctrines de Montesquieu et de Rousseau, et à l'hostilité vis-à-vis de l'idée que les actes des assemblées souveraines pouvaient être soumis à un contrôle des juges ${ }^{7 k}$. Mais $s^{\prime}$ il est vrai que la tradition démocratique française, à partir de 1789 , en matière de garantie des droits n'accepte pas un pouvoir judiciaire comme frein au pouvoir législatif, le projet de Sieyès de l'an III s'éloigne de cette conception. Sieyès proposa une procédure qui appartenait à l'ordre juridictionnel, quand l'idée dominante était que l'unique garantie des droits possible était à rechercher « dans le seul jeu des pouvoirs " ${ }^{79}$.

(75) «Tournant le dos à la tradition révolutionnaire, la constitution de l'an VIII confiait exclusivement la proposition des lois au gouvernement ", Michel PfrTUÉ, " La constitution de l'an VIIl ", op. cil., p. 286.

(76) Paul Bastid, Sieyès et sa pensée, op. cit., p. 445.

(77) «Bien plus ou bien autrement qu'une Cour constitutionnelle, ce jury est un gardien de la République ", Lucien JAUME, " Sieyès et le sens du jury constitutionnaire ", op. cit., p. 129.

(78) Voir Mauro CAPPELLETT, Il controllo giudiziario, op. cit., p. 81-84.

(79) Patrice ROLLAND, “La garantic des droits ", dans Jean BART, Jean-Jacques Clekre, Claude Courvolsier, Michel VerpauX (éd.), La constitution de l'an III, op. cit., p. 71. 
Larticle 21 de la constitution de l'an VIII ${ }^{\mathrm{x} 1}$, en continuité avec l'an III, imposa un contrôle de constitutionnalité des lois et des actes du gouvernement. Mais l'innovation de l'article 21 fut réduite par l'article 29 qui établissait (par rapport au Tribunat, organe propulseur vers le Sénat, organe qui jugeait) : « le voux qu'il manifeste en vertu du présent article n'ont aucune suite nécessaire et n'obligent aucune autorité constituée à une délibération ". C'était la négation du jury et la fin du projet de Sieyès, au moment où il semblait se réaliser ${ }^{81}$.

Le rôle du Sénat, à savoir contrôler le respect de la constitution et garantir sa supériorité par rapport aux autres normes par un contrôle préventif - article $37^{k_{2}}$-, restait théorique. Dans la pratique, la disposition de l'article 21 n'avait aucun effet : d'un côté, le Sénat ne pouvait pas décider tout seul et le Tribunat aurait hésité sur l'opportunité d'entrer en conflit avec le gouvernement; d'autre part aucun acte du gouvernement ne fut jamais l'objet d'un renvoi par le Sénat. "Sous le Consulat et sous les deux Empires, on institua un Sénat gardien de la Constitution. Mais ce gardien fut toujours entre les mains du premier consul et de l'empereur un instrument docile ${ }^{83}$.

Donc, le contrôle de constitutionnalité des lois - théorisé par Sieyès en l'an III et constitutionnalisé en l'an VIII -, partie importante et peutêtre la plus innovante de la constitution consulaire, n'a jamais fonctionné, et sa défaite représentait la première et la dernière tentative de créer en France un pouvoir neutre de nature politico-juridictionnelle ${ }^{x / 4}$.

Marco FIORAVANTI

Histoire du droit médiéval et moderne Université de Rome « Tor Vergata », Faculté de Droit, Via Bernardino Alimena, 5, 00173, Rome, Italie cocofioravanti@hotmail.com

(80) « Il [le Sénat conservateur] maintient ou annule tous les actes qui lui sont déférés comme inconstitutionnels par le Iribunat ou le Gouvernement ".

(81) Mario Battaglin, Contributi alla storia, op. cit., p. 66.

(82) "Tout décret du corps législatif, le dixième jour après son émission, est promulgué par le premier consul, à moins que, dans ce délai, il n'y ait cu recours au Sénat pour cause d'inconstitutionnalité. Ce recours n'a point lieu contre les lois promulguées ”.

(83) Ho Hio KY, Le contróle de la constitutionnalité des lois, op. cit., p. 185.

(84) Luigi LACCHE, « La garanzia della costituzione. Riflessioni sul caso francese ", Università degli studi di Macerata. Annali della facoltà di Giurispnudenza, 2000-2002, p. 41 et s. 